\title{
Investigación para la toma de decisiones de manejo en áreas marinas protegidas como la Isla del Coco, Costa Rica
}

\author{
Iria S. Chacón ${ }^{1 *} \&$ Fernando G. Soley ${ }^{2}$ \\ 1. Instituto Internacional en Conservación y Manejo de Vida Silvestre, Universidad Nacional, 1350-3000 Heredia, Costa \\ Rica; inatsalas@gmail.com \\ 2. Escuela de Biología, Universidad de Costa Rica, 2060 San José, Costa Rica; fgsoley@gmail.com \\ * Correspondence.
}

Recibido 27-II-2019. Corregido 16-VIII-2019. Aceptado 04-X-2019.

\begin{abstract}
Research for decision-making in marine protected areas such as Isla del Coco, Costa Rica. Introduction: Scientific information is often needed to guide management decisions, but marine protected areas usually lack such information. Further, these protected areas face the challenge of protecting highly-mobile pelagic species that move between protected areas in different countries and across fishing zones. In general, the dissociation that commonly exists between academic and conservation groups, which work under different objectives and reward systems, serves as an obstacle for producing the information that is needed by wildlife managers. This limitation is further enhanced in oceanic islands, such as Isla del Coco because of their mere remoteness, a condition that dramatically increases the economic and operational costs for doing research. Objective: To illustrate the challenge of generating useful scientific information for conservation decision making in protected areas, using Isla del Coco National Park in Costa Rica as a case study, and to propose possible solutions. Results: In order to produce the scientific information that these areas require, it is necessary to: i) distinguish between biologically relevant information, and information required for decision-making, ii) generate information about the threats to biodiversity, even in the absence of information about the species themselves, iii) establish clear goals and objectives for monitoring plans, and iv) build strong links between two types of groups: those that work from mainland and those that operate offshore; this includes working alongside fishing vessels. Conclusions: It will be a great challenge to articulate such relationships between groups, but this option seems more viable (in terms of associated logistic and economical costs) than attempting to collect the required data from an isolated academic platform. Also, this articulation appears to be the only way of generating information that is crucial for stock management, such as the accurate characterization of the fishing activity.
\end{abstract}

Key words: conservation research, biodiversity threats, biological monitoring, conservation politics, fishing overexploitation, pelagic species.

Chacón, I. S. \& Soley, F. G. (2020). Investigación para la toma de decisiones de manejo en áreas marinas protegidas como la Isla del Coco, Costa Rica. Revista de Biología Tropical, 68(Supl. 1), S1-S17.

A medida que se ha caracterizado y reconocido con mayor amplitud la magnitud de las amenazas a la biodiversidad, se ha vuelto necesario un manejo efectivo dentro y fuera de las áreas protegidas (Pullin, Knight, Stone, \& Charman, 2004). Las decisiones de manejo tienen un impacto directo sobre el recurso que se protege o utiliza, y por esto, se espera que sean tomadas con base en evidencia rigurosa (i.e., información científica) (Polasky, Carpenter, Folke, \& Keeler, 2011). Sin embargo, la información necesaria para la gestión a menudo no es suficiente o simplemente no existe, en especial, cuando se habla de áreas marinas protegidas (Lundquist \& Granek, 2005).

Una de las razones por las cuales no se cuenta con la información necesaria para la gestión, es que comúnmente, existe una 
disociación entre el sector académico y la gestión de las áreas protegidas (Gerber \& Schultz, 2001; Lundquist \& Granek, 2005). Esta disociación dificulta el proceso de diseñar investigación para la conservación, en parte porque el sector académico no conoce la información específica que se requiere, y también, porque el sector de gestión a menudo no comprende los alcances y limitaciones de las investigaciones que se realizan en las áreas protegidas (Huenneke, 1995; Laurance et al., 2012). Aun cuando se superan estas barreras, el sistema de recompensas del sector académico, donde comúnmente se premia los estudios a corto plazo, dificulta generar datos a largo plazo que son útiles para el manejo de áreas protegidas (Verner, 1992; Magurran et al., 2010).

Otro obstáculo para la generación de información para la gestión es que, comúnmente, los únicos académicos asociados a las áreas protegidas son los biólogos o afines (Ellstrand et al., 2010). Aunque este acercamiento es loable, lastimosamente, mucha de la información que se requiere se aleja del perfil "tradicional" de un biólogo académico. La gestión de las áreas protegidas depende del conocimiento detallado sobre las amenazas que afectan a la biodiversidad, y estas amenazas son, en su mayoría, de origen antropogénico (Salafsky, Salzer, Ervin, Boucher, \& Ostile, 2003; Ellstrand et al., 2010). Por lo tanto, una adecuada caracterización de estas amenazas necesita de esfuerzos interdisciplinarios (Newing, 2011).

En el caso de las áreas marinas protegidas, es común que se necesite información sobre actividades extractivas (e.g., pesca legal e ilegal), y esto hace necesario un trabajo interdisciplinario que involucre tanto a profesionales de las ciencias biológicas como a profesionales de las ciencias sociales (Laurance et al., 2012). En ocasiones, lo interdisciplinario necesitará extenderse aún más para poder recopilar la información requerida. Por ejemplo, para caracterizar ciertas amenazas como la pesca ilegal, es necesario trabajar de la mano con los funcionarios del área protegida (p.ej. López-Garro, Zanella, Martínez, \& Golfín-Duarte, 2016), la autoridad pesquera, guardacostas, u otros, debido a que la obtención de esta información elude las habilidades, entrenamiento, y/o disposiciones de profesionales académicos. El trabajo interdisciplinario también puede ser esencial en generar datos biológicos (p.ej., biomasa, abundancias relativas de especies), especialmente en sitios que son más frecuentados por personas no académicas (Magurran et al., 2010; Hyder, Townhill, Anderson, Delany, \& Pinnegar, 2015).

Antes de pensar en el tipo de información que se requiere para mejorar la efectividad de manejo en áreas marinas protegidas (y cómo recopilarla), es conveniente aclarar algunos aspectos. Esperamos que estas aclaraciones sean de utilidad para las personas no académicas que trabajan en conservación, y para los académicos que tienen como objetivo lograr impactos positivos en conservación. En este ensayo, comentamos brevemente sobre: i) la necesidad de distinguir entre información biológica e información requerida para la gestión (una distinción que no es obvia); ii) la importancia de generar información sobre amenazas a las especies (aún en ausencia de datos sobre las especies); iii) las ventajas de orientar claramente los planes de monitoreo; y iv) algunos de los retos al articular esfuerzos existentes para generar la información requerida. Para ilustrar algunos puntos, utilizamos como referencia al Parque Nacional Isla del Coco (PNIC) en Costa Rica, un sitio que comparte desafíos con muchas otras áreas protegidas, en especial, con aquellas del Pacífico Tropical Oriental.

\section{La investigación para la toma de decisiones}

Como punto de partida, conviene recalcar que existe una diferencia entre la "investigación científica básica" y la "investigación para la gestión". La investigación científica se realiza con el fin de generar conocimiento, y por lo tanto posee un valor intrínseco para la ciencia, independientemente de si responde o no a necesidades de conservación (Primack, 2006). Por otro lado, la investigación que se realiza con el fin de generar información necesaria para la 
toma de decisiones en conservación responde a una agenda muy diferente de prioridades, que es muy dependiente del contexto específico en que se realiza (Primack, 2006; Newing, 2011). Así, la investigación para la gestión representa solamente un componente dentro de la gran gama de preguntas científicas; y es natural que los objetivos, público meta, aspectos espaciales y temporales, así como los temas de estudio difieran entre estas dos categorías generales de investigación científica (Huenneke, 1995).

¿Cuál es la necesidad de distinguir entre investigación científica "básica" e investigación para la conservación? Por un lado, esto ayudará a los profesionales en conservación a definir puntualmente la información que necesitan. Por otro lado, este ejercicio ayudará a los investigadores interesados en conservación a definir el impacto de su investigación en ese sentido. Uno de los puntos más importantes tiene que ver con la disponibilidad de recursos que tenemos para realizar conservación. La realidad es que la lista de especies, hábitats, y ecosistemas amenazados es cada vez mayor, y no disponemos de todos los recursos económicos/logísticos necesarios para hacer frente a todos los problemas de conservación (Possingham, Andelman, Noon, Trombulak, \& Pulliam, 2000; Bottrill et al., 2008). Por lo tanto, en conservación, es importante canalizar los recursos en la dirección que impera; lo cual, aunque parezca obvio, en la práctica no lo es. De hecho, es común escuchar sobre investigaciones en áreas protegidas que son interpretadas erróneamente como investigaciones para la conservación.

Uno de los puntos más críticos en este sentido (y que pueden practicar las personas encargadas de la gestión o académicos) es anticiparse a los resultados de las investigaciones que se pretende realizar, y plantearse la utilidad de los resultados que se van a obtener en el contexto del área protegida. A menudo, como investigadores en biología, al momento de justificar nuestra investigación, nos dejamos llevar por lo maravilloso de nuestra especie de estudio (la cual a menudo se encuentra en riesgo de extinción), o lo cautivante del sistema, o el sentimiento especial que nos genera un área protegida. Esto, no nos permite darnos cuenta de que la investigación que es prioridad para conservación, en muchos casos, es distinta, y se enfoca en áreas degradadas, ambientes poco prístinos, especies menos carismáticas, o en temáticas sociales, que como biólogos nos cuesta enfrentar.

Cabe mencionar que no existe una línea clara que divida el proceso científico de generación de conocimiento en investigación "básica" o investigación "aplicada" (Soulé, 1985). Es de esperar que la utilidad de una investigación biológica a la conservación se presente en una escala de grises. Por ejemplo, el estudio de una especie en peligro crítico de extinción que habita un área protegida no necesariamente generará información de utilidad para proteger sus poblaciones. Igualmente, el estudio de una comunidad de tiburones que habita un área protegida no necesariamente identificará las razones por las cuales fluctúan las abundancias relativas de cada especie (ver abajo). En ambos casos, se están estudiando especies amenazadas, por lo que se podría pensar que se trata de investigación aplicada, pero la utilidad de la información que se genere para la gestión dependerá casi que exclusivamente del enfoque del estudio. Aunque todo el conocimiento de especies amenazadas es de interés para la conservación (entre más conozcamos sobre las especies, más podremos anticiparnos a las consecuencias de las amenazas o a las decisiones de manejo); lo cierto es que, para el manejo, habrá información que será más útil que otra.

Es de esperar que esta escala de grises entre investigación "básica" o "aplicada" genere confusión al momento de i) definir las prioridades de investigación de un área protegida, y de ii) interpretar los resultados de las investigaciones y decidir cómo utilizarlos para la gestión del área protegida. Sin embargo, en un contexto inminente de amenazas, la distinción entre investigación "básica" e investigación para la toma de decisiones se hace más evidente. Lo anterior, debido a que la contribución de la investigación (para la conservación), en este contexto, debería de estar orientada a generar 
datos que permitan tomar decisiones de manejo que tengan un impacto directo en la reducción de las amenazas (Laurance et al., 2012).

En este sentido, cuando se quiere definir la investigación requerida para el manejo de un área protegida, conviene antes priorizar las amenazas que atentan contra la biodiversidad o ecosistemas de dicha área (Salafsky, Margoluis, \& Redford, 2002; Salafsky et al., 2003). Esto hará más fácil el ejercicio de discernir cuál es la investigación "aplicada" que se requiere. Si se tiene claridad sobre la información que se necesita, entonces se facilitará el adelantarse al tipo de resultados que generarán distintas investigaciones y evaluar si estas producen los resultados requeridos. Una vez que se haga conciencia sobre el tipo de resultados que se requieren, se facilitará entonces el definir líneas de investigación claras.

Finalmente, es importante resaltar que la investigación "básica", además de poseer un valor intrínseco para la ciencia y la sociedad, también es de importancia para el área protegida por diversas razones. Por ejemplo, la información científica que se genera bajo esta modalidad, es de interés para el público general, de manera que puede ser utilizada para atraer visitantes al área (incluso a otros científicos), o para hacer divulgación de las maravillas biológicas que se protegen en el área. También, esta información puede permitirle al área protegida demostrarle a la comunidad (nacional/internacional) que se está cumpliendo con los objetivos para los cuales fue creada (p.ej., el proteger ciertas especies, ecosistemas, o fenómenos naturales). Incluso, a partir de investigación "básica" puede surgir información que de forma inesperada resulte de interés para el sector gestión (p. ej., en la Isla del Coco, la presencia de contaminación por microplásticos en los fondos marinos se descubrió en un estudio enfocado en la taxonomía de los organismos que ocupan estos hábitats; Cortés com. pers.).

La idea de distinguir entre investigación "básica" e investigación "aplicada" no es la de excluir la investigación "básica" de un área protegida, o mucho menos. La información que genera la investigación "básica", debería de ser siempre bienvenida por el área protegida. En parte, por las razones mencionadas anteriormente, y en parte, porque al promover una relación más estrecha entre el sector académico y el sector gestión, se genera un ambiente más favorable para articular esfuerzos y generar información de mayor relevancia para la gestión. Dicha articulación, es un elemento estratégico para elevar la capacidad de manejar riesgos, amenazas y presiones, puesto que la incidencia de estos factores se minimiza a medida que se formen más alianzas alrededor de un objetivo en común. Entonces, el esfuerzo de discernir cuál es la investigación "aplicada" debería de tener como objetivo, definir cuál es la información que requiere con mayor urgencia el área protegida para reducir las amenazas sobre los ecosistemas o especies que protege. Debido a que los objetivos de conservación de cada área protegida son distintos (y también lo son las amenazas que atentan contra el cumplimiento de dichos objetivos), la definición de qué es investigación "aplicada" debería de estar supeditada al contexto de cada área protegida.

\section{¿Qué se requiere? \\ ¿Investigación de especies 0 investigación sobre amenazas a las especies?}

El propósito fundamental de la investigación para la conservación es el de informar e incidir en las políticas de conservación (Robertson \& Hull, 2001). La investigación biológica ha sido efectiva en responder ciertas preguntas de importancia para la conservación; por ejemplo, se tiene una mejor idea de cuáles especies y ecosistemas están amenazados o en peligro de extinción (Salafsky et al., 2002). También, se han hecho esfuerzos significativos en priorizar especies y ecosistemas que requieren atención en términos de conservación, a través de tendencias, estatus taxonómico, endemismo, ámbito geográfico, utilidad económica, entre otros (Nichols \& Williams, 2006). Adicionalmente, muchos estudios científicos han ayudado a identificar problemas de conservación en áreas protegidas existentes. Por ejemplo, 
con relación a las áreas marinas protegidas, sabemos que las especies pelágicas de interés comercial son explotadas en diferentes países, lo cual complica el controlar las tasas de cosecha de estas especies (Laurance et al., 2012). Por lo tanto, la investigación para la conservación también debería de estar enfocada en buscar soluciones a esos problemas, y no solamente en continuar priorizando especies, hábitats y ecosistemas para conservar (Nichols \& Williams, 2006).

No hay duda de que la conservación necesita de información que se genera a través de investigaciones biológicas; sin embargo, también es imperante integrar otro tipo de información para generar e implementar medidas de conservación efectivas (Balmford \& Cowling, 2006; Newing, 2011). En la mayoría de los casos, la conservación de la biodiversidad se ve amenazada por actividades humanas (Salafsky et al., 2002). Entonces, para proteger la biodiversidad, necesitamos que la investigación incorpore información detallada sobre los factores que están causando estas amenazas. Quizás, el mayor esfuerzo en ese sentido a nivel global se ha hecho para caracterizar la amenaza del calentamiento global; sin embargo, a menudo existen otros factores locales que producen amenazas más inmediatas o de mayor magnitud. Por lo general, dichas amenazas están claramente identificadas por los funcionarios de las áreas protegidas o integrantes de otras instituciones $\mathrm{u}$ organizaciones no gubernamentales (ONG) que laboran en el sitio. Sin embargo, el detallar estas amenazas (p.ej., distribución espacial, frecuencia, intensidad, sectores involucrados, causas subyacentes) requerirá de esfuerzos conjuntos entre el sector académico y el sector gestión - ver sección de Retos para la articulación de esfuerzos entre distintos sectores. La necesidad de realizar investigaciones sobre las amenazas se puede ilustrar utilizando como ejemplo el estudio de tiburones y rayas en la Isla del Coco.

\section{Amenazas a tiburones y rayas en la Isla del Coco}

Gracias al sector turismo, el Parque Nacional Isla del Coco cuenta con el monitoreo acuático visual más extenso (1991-presente) que se ha hecho para tiburones y rayas a nivel global; este esfuerzo tan extenso de monitoreo es único en el Pacífico Tropical Oriental (White, Myers, Flemming, \& Baum, 2015). Los datos son tomados durante cada inmersión por los guías de buceo, y aunque existan limitantes, esta investigación "informal" pero consistente tiene la ventaja de aprovechar las observaciones de las personas que pasan mayor tiempo en el agua buscando a estos animales. De hecho, los datos obtenidos en este monitoreo a cargo del sector turismo han permitido documentar tendencias espacio-temporales para las poblaciones de varias especies de tiburones y rayas (Sibaja-Cordero, 2008; White et al., 2015).

Estos estudios mostraron una disminución en el tamaño de la población para varias de las especies que se monitorean, y sobre todo para especies pelágicas de interés comercial (Sibaja-Cordero, 2008; White et al., 2015). Lo más probable es que el decline de estas especies haya sido causado por la pesca ilegal y la sobrepesca fuera de los límites del área protegida (Sibaja-Cordero, 2008; White et al., 2015; Arias \& Pressey, 2016). Sin embargo, caracterizar el impacto de la sobrepesca en estas poblaciones se dificulta debido a que, en parte, las fluctuaciones temporales en el tamaño de las poblaciones o en la presencia de determinada especie, también se ven afectadas por otros factores, como por ejemplo, las oscilaciones climáticas (Sibaja-Cordero, 2008; White et al., 2015). En general, las fluctuaciones poblacionales son algo común en ecología, y tienen componentes determinísticos (elementos predecibles) y estocásticos (elementos aleatorios), que operan simultáneamente (Lande, Engen, \& Sæther, 2003). Por lo tanto, encontrar los componentes determinísticos y poder diferenciarlos de elementos estocásticos resulta sumamente complejo en poblaciones naturales.

Un elemento crucial para distinguir el efecto de un componente determinístico es, por supuesto, cuantificar ese componente, y ver cómo se relaciona con la variable respuesta (p.ej., abundancia relativa de una especie). En el caso particular de la Isla del Coco, si se 
pudiese caracterizar la amenaza de sobrepesca (p.ej., en intensidad, distribución espacial y temporal), entonces se podría entender de mejor manera cómo se relaciona esta amenaza con la dinámica poblacional de las distintas especies de tiburones y rayas. Así, la caracterización de la amenaza de pesca permitiría diferenciar con mayor claridad entre el efecto que esta produce directamente y el efecto que producen las oscilaciones climáticas (u otros factores) en distintos parámetros poblacionales o de distribución de estas especies.

Una ventaja clara de caracterizar la amenaza a nivel espacial y temporal es que esto permitiría concentrar las acciones de manejo en sitios y momentos prioritarios (p.ej., patrullajes o regulaciones de uso del recurso), y así lograr mayor impacto de conservación. De hecho, la utilidad de la información sobre amenazas para tomar decisiones de manejo es tal que, se puede justificar la generación de esta información aún en ausencia de "datos biológicos" (i.e., datos tomados directamente sobre las especies). Por ejemplo, en áreas marinas de protección absoluta, donde no se permite la extracción del recurso, pareciera más importante documentar con precisión dónde y cuándo se efectúa la pesca ilegal, para realizar labores de patrullaje más efectivas, que el intentar documentar cómo la pesca ilegal afecta las tendencias poblacionales de determinadas especies. Se debe notar que, por el contrario, la última pregunta si adquiere prioridad en áreas protegidas donde se permite un manejo del recurso; tal es el caso de las áreas de pesca responsable en las que resulta fundamental la toma de datos de las capturas.

La razón por la cual se recomienda enfocar la investigación en las amenazas, aún en ausencia de datos sobre las especies, es porque lo que se quiere es precisamente reducir las amenazas, y no tanto documentar el impacto de estas sobre las especies (la documentación del impacto fue lo que llevó, usualmente, a la creación del área protegida). Luego de la creación de un área protegida donde se prohíbe la extracción, el objetivo primordial ya no es seguir documentando el impacto negativo sobre la especie (esto ya se determinó), sino documentar que efectivamente se están reduciendo las amenazas en ese sitio. Cuando se caracteriza la amenaza, aún en ausencia de datos biológicos, se pueden guiar acciones de manejo, pero no se puede decir lo mismo cuando ocurre lo inverso (cuando existe solamente información biológica). La instalación reciente de un radar en la Isla del Coco para monitorear el ingreso de embarcaciones al área marina protegida es un avance importante en esta dirección. El radar, además de desincentivar el ingreso al área, también ha permitido documentar tendencias espaciotemporales en el ingreso ilegal de embarcaciones, y esta información se planea utilizar para guiar los patrullajes de los guardaparques (Maikel Pérez, com. pers.).

Un punto clave es que, aun cuando se requiere información biológica, la misma investigación sobre amenazas a las especies puede también generar esta información. Por ejemplo, los datos de captura pesquera se pueden analizar según el esfuerzo pesquero, y utilizar esta información para generar índices de abundancia relativa y patrones espaciotemporales de distribución (i.e., aproximar los esfuerzos pesqueros a esfuerzos de monitoreo) (Camhi, Pikitch, \& Badcock, 2018; pero ver Maunder et al., 2006). También, se puede llevar un registro de los volúmenes de captura por embarcación, las tallas de los individuos capturados, y las tallas a las que se adquiere la madurez sexual, para detectar a qué niveles de extracción se está comprometiendo la viabilidad del recurso pesquero a largo plazo (Trippel, 1995; Law, 2000). La opción de obtener información a partir de la actividad extractiva es llamativa, ya que es más factible su implementación desde el punto de vista económico. Como la extracción de recursos es una actividad autofinanciada, es más viable obtener información biológica por este medio, que mediante monitoreos biológicos financiados por el Estado o el sector conservación. Lo anterior es evidente en sitios tan alejados de tierra firme, como las islas oceánicas, en donde los costos logísticos y económicos (además de los riesgos laborales) de un monitoreo efectivo son extremadamente elevados; en especial si se realizan a largo plazo. 
Así, este tipo de actividad puede generar tanto información biológica, como información de la propia actividad (i.e., amenaza al recurso), que es precisamente la información que se requiere cuando se decide manejar un recurso.

Debido a las dificultades logísticas y económicas, el implementar un monitoreo biológico (ya sea basado en observaciones visuales, o incluso mediante sensores remotos) en altamar (i.e, Zona Económica Exclusiva) se vuelve un sueño aparentemente imposible. Por el contrario, parece ser más factible el generar información biológica a partir de las mismas actividades extractivas que operan bajo el marco legal existente (por supuesto esto trae consigo otros retos - ver sección de Retos para la articulación de esfuerzos entre distintos sectores). La dificultad de obtener datos en altamar se ve reflejado en el hecho de que se ha generado muy poca información acerca de la mayoría de las especies pelágicas que se encuentran en la Isla del Coco (Camhi et al, 2018). Por ejemplo, en todo el Pacífico Tropical Oriental no se tiene información poblacional (generada por estudios biológicos) para el tiburón sedoso (Carcharhinus falciformis), aun cuando se sabe que esta especie de tiburón es la que sufre de mayor captura incidental en las pesquerías de atún de cerco, y que también es objetivo de pesca dirigida para obtener sus aletas (Rojas et al., 2000; Watson, Essington, Lennert-Cody, \& Hall, 2009; Arias, Pressey, Jones, Álvarez-Romero, \& Cinner, 2014; López-Garro et al., 2016). Este escenario se repite para muchas otras especies pelágicas sometidas a una fuerte presión pesquera; por ejemplo, el atún aleta amarilla (Thunnus albacares) (Whoriskey, Arauz, \& Baum, 2011; Dapp, Arauz, Spotila, \& O'Connor, 2013). La escasez de información científica acerca de estas especies imposibilita determinar con exactitud cómo la pesca está impactando sus poblaciones (Arias et al., 2014; GonzálesAndrés, Lopes, Cortés, Sánchez-Lizano, \& Pennino, 2016; López-Garro et al., 2016). Sin embargo, el impacto sobre las poblaciones sí se podría estimar si se analizara la información del éxito de captura y extracción de individuos con relación al esfuerzo de captura (controlando por las técnicas utilizadas).

Esta información podría ser generada por las flotas pesqueras que operan bajo el amparo legal, ya que el costo asociado es muchísimo menor (p.ej., observadores a bordo, reportes obligatorios de volúmenes de captura) que el de organizar una expedición de monitoreo independiente. Aunque reconocemos que la articulación de esfuerzos con este sector no será una tarea para nada fácil (y que requerirá quizás de cambios en la normativa existente), creemos que la alternativa de generar monitoreos efectivos en altamar y a largo plazo de forma independiente, aparenta ser, por el contrario, una tarea imposible. Además, aún si se superara la barrera económica/logística y se lograra realizar monitoreos a largo plazo de forma independiente al sector pesquero, terminaríamos, una vez más, con el problema de no contar con una caracterización adecuada de la amenaza (p.ej., cuotas extraídas, distribución espacial y temporal de la actividad), y, por ende, seguiríamos siendo incapaces de relacionar la intensidad de la amenaza a las tendencias poblacionales de estas especies. Al parecer, no se puede escapar de la necesidad de trabajar en conjunto con el sector pesquero para garantizar la viabilidad del recurso a largo plazo (Law, 2000).

Por último, otra ventaja de caracterizar las amenazas en intensidad, frecuencia, y distribución espacio-temporal, es que esta información se puede analizar a la luz de las distintas acciones de manejo que ha implementado el área protegida. Esto permitiría demostrar el impacto o relevancia de estas acciones para lograr los objetivos de conservación del área protegida (p.ej., patrullajes, instalación de radares, etc.), y así determinar cuáles acciones mantener y replicar o cuales acciones necesitan modificarse. Lo anterior es un componente crucial del manejo adaptativo (Bakker \& Doak, 2009), y funciona además para demostrarle a la sociedad civil el impacto en conservación que se está teniendo, en relación a los recursos económicos y logísticos que se están implementando (Parrish, Barun, \& Unnash, 2003; Fox et al., 2012). 


\section{Ventajas de dirigir el monitoreo biológico}

El monitoreo biológico es parte integral del manejo adaptativo, mediante el cual se pretende conocer el estado del sistema ecológico, y medir los impactos de las medidas de gestión (ver Legg \& Nagy, 2006; Nichols \& Williams, 2006). Sin embargo, los esfuerzos de monitoreo a menudo carecen de un trasfondo conceptual y se convierten en lo que se conoce como "monitoreos de vigilancia", los cuales carecen de objetivos claros y terminan siendo una pérdida de recursos económicos y de tiempo (Vos, Meelis, \& Keurs, 2000; Nichols \& Williams, 2006). En parte, esto se debe a que los monitoreos de vigilancia asumen que es fácil atribuir los cambios obervados (p. ej., en presencia/ ausencia de especies, o en adundancias relativas de especies), a causas particulares.

En realidad, es difícil atribuir variaciones ecológicas a factores específicos. Desde el punto de vista de gestión, parece ser poco productivo el enfocarse en detectar "cambios en los ecosistemas"; ya que por un lado, los ecosistemas no se comportan como entes individuales y estáticos, y por otro, lo común es que una perturbación no afecte a todas las especies, o no lo haga por igual (ver Lawton 1999; Ricklefs, 2008). Esto dificulta o imposibilita el poder delegar el monitoreo de una comunidad biológica al monitoreo de ciertas especies que "la representen" o "indiquen su estado de salud" (p.ej., depredadores tope) (ver Sibert, Hampton, Kleiber, \& Maunder, 2006; y Ricklefs, 2008). Una alternativa es entonces, dirigir los esfuerzos de monitoreo a las especies que están siendo particularmente afectadas.

Sin embargo, aun cuando los monitoreos se enfoquen en estudios poblacionales de ciertas especies, uno de los mayores retos del monitoreo, es poder distinguir si un cambio se puede atribuir a un factor "externo" (i.e., actividades antropogénicas), o si se trata de un cambio "natural" (Magurran et al., 2010; ver también Anderson et al., 2008). Lo anterior, debido a que lo común es que las poblaciones biológicas fluctúen en el tiempo de forma natural (Parrish et al., 2003). El atribuir causas de decline se dificulta aún más en los casos en que hay explotación pesquera, debido a que las poblaciones experimentan mayores fluctuaciones en su abundancia (Anderson et al., 2008). $\mathrm{Si}$ aun en estudios ecológicos dirigidos explícitamente a comprender las fluctuaciones en las poblaciones, ha sido difícil discernir el efecto de factores antropogénicos de los factores naturales (p.ej., oscilaciones climáticas) (ver Anderson et al., 2008), se espera que en esfuerzos de monitoreo amplios (como el monitoreo de vigilancia), sea más difícil o imposible responder a este tipo de preguntas. Por un lado, el detectar tendencias poblacionales puede llevar años de recolección de datos, tanto así que los cambios negativos pueden llegar a detectarse cuando ya es demasiado tarde para hacer algo (Nichols \& Williams, 2006). Por otro lado, el poder estadístico necesario para detectar tendencias poblacionales con claridad se ve afectado por muchos factores; por ejemplo, la duración de las series temporales del muestreo, la precisión de las medidas, la cantidad de sitios de muestreo, y la frecuencia de los muestreos (Magurran et al., 2010).

Además, se critica que el monitoreo se enfoca en detectar tendencias, pero los temas cruciales relacionados con las causas y la atención de las tendencias negativas no se abordan (Nichols \& Williams, 2006). El monitoreo no solo debe ir enfocado en detectar cambios, sino en entender las causas de ese cambio, para poder encontrar la manera de atender o remediar cambios negativos (Parrish et al., 2003; Nichols \& Williams, 2006). Es decir, el monitoreo debería de ayudar a guiar estrategias de conservación activas, mediante el entendimiento de cómo se pueden manipular variables para mejorar las condiciones de una población o ecosistema (Nichols \& Williams, 2006).

\section{El monitoreo supeditado a la gestión y no solo a la biodiversidad}

Un programa de monitoreo debe empezar con la identificación del tipo de información que requiere el sector político-administrativo para tomar las decisiones de gestión más 
adecuadas (Vos et al., 2000). Así, el monitoreo puede dejarse de percibir como una herramienta que, por sí sola, descubrirá y guiará las acciones de manejo (Legg \& Nagy, 2006; Nichols \& Williams, 2006). Por el contrario, el monitoreo debe de tener objetivos claros y estar enmarcado en un panorama de gestión, en donde se tiene claridad de antemano sobre cuáles datos se necesitan, el porqué de estos datos, y cómo interpretarlos (Vos et al., 2000). Sin embargo, muchos de los monitoreos que se realizan en las áreas protegidas tienen un enfoque meramente descriptivo, y se dedican a observar qué ocurre con las poblaciones de las especies o los ecosistemas, y dejan de lado la caracterización de las amenazas. Lo anterior, lleva a olvidar el fin último de un monitoreo: generar información que permita orientar decisiones de manejo. Por lo tanto, es importante no solo monitorear la biodiversidad, sino también las variables que podrían ser manipuladas en procesos de conservación activos; y lo segundo es más factible de hacer que lo primero.

El Pacífico Tropical Oriental es una de las regiones marinas más importantes en términos de biodiversidad y especies endémicas (Robertson \& Cramer, 2009), y también en recurso pesquero (p.ej. atunes y marlines) (Hinton et al., 2014). Entonces, es importante que, a la hora de desarrollar estrategias de monitoreo en esta región, se tomen en consideración ambos factores: biodiversidad y recurso pesquero. Por un lado, los servicios ecosistémicos, como la pesca, son mejor soportados por comunidades biológicas diversas (Hector \& Bragchi, 2007). Y, por otro lado, pensar en monitoreos que tienen que ver con especies objetivo de pesca podría crear mayor interés económico y político sobre estos programas, ya que es un tema que no solo interesa a los biólogos, sino que es de interés público (especialmente para aquellos interesados en que se mantenga la actividad a largo plazo).

Por ejemplo, en la Isla del Coco, el monitoreo biológico de especies pelágicas debería de estar dirigido, principalmente, a taxones que son objetivo de pesca o que son directamente afectadas a través de la pesca incidental (p. ej., atún aleta amarilla - Thunnus albacares, tiburón sedoso - Carcharhinus falciformis, tiburón martillo - Sphyrna lewini) (Hinton et al., 2014). Dicho monitoreo debería de ir acompañado de información sobre las amenazas (i.e., principalmente la explotación pesquera y la pesca ilegal), para lograr desarrollar modelos predictivos que indiquen cómo se impacta el recurso pesquero y la diversidad marina (a corto y largo plazo) bajo distintos escenarios de extracción en la Zona Económica Exclusiva. Este tipo de monitoreo de especies, que son directamente impactadas por la amenaza, permitiría establecer relaciones de causalidad con mayor facilidad que, por ejemplo, monitoreos de especies carismáticas que están relacionadas principalmente de forma indirecta con la amenaza (p.ej., a través de redes tróficas).

Por último, estos esfuerzos de monitoreo (principalmente aquellos relacionados a las amenazas) deberían de ampliarse para incluir otras zonas del Pacífico Tropical Oriental, ya que se trata de especies pelágicas con alta movilidad. Los amplios rangos de desplazamiento de estas especies hacen que no sea fácil interpretar lo que pasa en un sitio si no se conoce lo que pasa en los otros sitios. Una posibilidad para lograr este objetivo tan ambicioso es trabajar de la mano con el sector de extracción. Esto permitiría generar información biológica e información de las cuotas de aprovechamiento y descarte por parte de este sector. Por supuesto, esto trae consigo grandes retos, principalmente de índole política (ver sección Articulación entre el sector conservación y el sector de extracción).

En conclusión, al diseñar estrategias de monitoreo se debe de tomar en cuenta los objetivos del programa, los objetos de estudio, las variables a monitorear, el diseño experimental, los horizontes de tiempo mínimos, y el mantenimiento y organización de los datos (Vos et al., 2000). Lo anterior quiere decir que, antes de comenzar cualquier monitoreo es necesario una articulación entre el sector académico y 
el de gestión, ya que ambos deberán asumir diferentes papeles dentro de las estrategias de monitoreo.

\section{Retos para la articulación de esfuerzos entre distintos sectores}

\section{Articulación entre el sector académico y el sector conservación}

Una amplia comunicación entre productores y usuarios del conocimiento es esencial para el desarrollo de soluciones creíbles, legítimas, y relevantes para los problemas de conservación (Sutherland et al., 2011). Sin embargo, lograr una comunicación adecuada entre el sector gestión y el sector académico representa, a menudo, un reto. Los temas de investigación prioritarios para la academia son variados y a menudo no son congruentes con la información que requieren los tomadores de decisiones en conservación en su práctica diaria (Sutherland, Fleishman, Mascia, Pretty, \& Rudd, 2011; Karam-Gemael, Loyala, Penha, \& Izzo, 2018). Esta falta de concordancia entre sectores, puede tener consecuencias negativas sobre la conservación de la biodiversidad (Karam-Gemael et al., 2018).

Los científicos y los profesionales en conservación difieren en sus objetivos profesionales, restricciones logísticas, y sistemas de recompensa (Huenneke, 1995). Para el científico académico, el éxito de su carrera profesional depende de publicar los resultados de sus estudios en revistas científicas, obtener financiamiento, y proveer buenas oportunidades de entrenamiento para sus estudiantes (Huenneke, 1995), independientemente de si su orientación académica tiene que ver o no con áreas aplicadas de la ciencia (p.ej., la conservación en el caso de la Biología). Debido al interés en publicar constantemente, y a las dificultades de obtener financiamiento a largo plazo, es común que en el sector académico, se premie los estudios a corto plazo, mientras que, para el manejo de áreas protegidas, son aún más necesarios los estudios a largo plazo (Verner, 1992; Magurran et al., 2010). Por su parte, el profesional que trabaja en conservación está acostumbrado a resolver problemas específicos de manera costo-efectiva y con rapidez, así que es natural que este profesional desee respuestas concretas a preguntas específicas, para poder guiar esfuerzos de conservación (Huenneke, 1995).

Aunque los objetivos profesionales del sector académico y el de gestión sean diferentes, existen mecanismos por los cuales se puede promover una colaboración entre ambos grupos. Uno de los mecanismos que se puede utilizar, es identificar en conjunto prioridades de investigación para el área protegida. De esta manera, las prioridades serán atractivas para algunos profesionales del sector académico, y de utilidad para las personas que hacen conservación (Sutherland et al., 2011). Por un lado, el sector académico puede entender cómo hacer su investigación más relevante para la práctica de la conservación (Karam-Gemael et al., 2018); lo cual puede contribuir en acceder a diferentes fuentes de financiamiento, crear más oportunidades para los estudiantes, y lograr una mayor divulgación sobre sus investigaciones (Huenneke, 1995). Por otro lado, los profesionales en conservación podrían respaldar sus decisiones con evidencia científica, además de obtener perspectivas más amplias acerca de la conservación (Karam-Gemael et al., 2018).

\section{Necesidad de articular esfuerzos en áreas remotas}

Es importante señalar algunas características que presenta la Isla del Coco, que se traducen en dificultades, tanto para la gestión como para hacer investigación. Estas características no son exclusivas del sitio, sino que se comparten con muchas otras áreas marinas protegidas (Fox et al., 2012; Ward-Paige, 2017). Principalmente, estas son: i) su condición de remotidad (a $\sim 550 \mathrm{~km}$ de tierra firme), alejada de los centros operativos del Estado, sector académico y ONG; y ii) el hecho de proteger especies marinas pelágicas, que entran y salen del área protegida y que se desplazan entre las jurisdicciones de varios países (p.ej. Costa Rica, Colombia, Ecuador y Panamá). En esta sección, comentamos brevemente sobre cómo 
estas características determinan las pautas para hacer investigación; comenzamos con la condición de remotidad.

Para realizar investigación para la toma de decisiones en el ámbito marino, en áreas tan remotas como La Isla del Coco, se necesita de una coordinación muy precisa entre dos grupos de personas: las que están en el sitio (y que se dedican principalmente a labores no académicas), y las que pasan la mayoría de su tiempo lejos del sitio, pero que trabajan en investigación. El perfil profesional del segundo grupo de personas (i.e., personal académico y de ONG) es más o menos homogéneo, mientras que el del primero puede ser muy variado; por ejemplo, funcionarios del área protegida, sector turismo, guardacostas, o flotas pesqueras. Por lo tanto, la efectiva coordinación entre estos grupos va a necesitar de procesos específicos para cada área protegida. Las personas que están en el sitio representan un motor para la generación de datos, mientras que el sector académico es un aliado indispensable para diseñar y definir los objetivos de la investigación, así como para el análisis, interpretación, y almacenamiento de los datos que se generen (ver Bonney et al., 2014).

Dicha coordinación entre sectores es un requisito desde el punto de vista logístico, pero también puede ser la única forma de recopilar cierta información. Por ejemplo, la integración de personas locales (generalmente de perfil no-académico), en el proceso de investigación adquiere relevancia particular cuando la información que se necesita es a largo plazo (i.e., monitoreos). Aún si el sector académico está interesado y puede financiar expediciones costosas de monitoreo, la resolución temporal de esta información será pobre en comparación a la información que pueden generar las personas que están continuamente en el sitio (ver Bonney et al., 2014). Sin embargo, existe una razón más importante para estimular una relación estrecha entre el sector gestión y el sector académico; y es que a menudo, esta será la única forma de generar información detallada sobre las amenazas que atentan contra la biodiversidad y ecosistemas del área protegida.
Las personas que generan información acerca de las amenazas, son casi exclusivamente aquellas que forman parte del personal de las áreas protegidas (p.ej. guardaparques, guardacostas, policía, o funcionarios equivalentes). Lo anterior, debido a que el sector académico (en su mayoría), trabaja con las especies del área protegida, y no con las amenazas. El perfil profesional de los académicos raramente tiene que ver con actividades diarias de patrullajes y decomisos, o el abordaje de embarcaciones pesqueras. Aunque existan sensores remotos para el monitoreo de algunas amenazas (p.ej., actividad pesquera), la precisión que se requiere a menudo va en otros sentidos (p.ej., decomisos, toneladas capturadas para distintas especies, porcentaje de descarte de dicha captura, tallas, etc.), y es información que los sensores remotos no pueden brindar.

\section{Ejemplos de integración de esfuerzos en la Isla del Coco}

En este sentido, en el Parque Nacional Isla del Coco existen algunos casos positivos de integración de sectores para la generación de información. Por ejemplo, el estudio de LópezGarro y colaboradores (2016) caracterizó la distribución espacial y temporal de la pesca ilegal en la Isla del Coco, además de la composición y porcentajes de especies capturadas, gracias a un esfuerzo conjunto entre funcionarios del área protegida (i.e., encargados de las labores de control y vigilancia) y biólogos (López-Garro et al., 2016). En este caso, las labores de protección y vigilancia, en conjunto con un acompañamiento científico, permitieron que se diera un monitoreo con un objetivo claro y que esta información se analizara apropiadamente y se publicara en un artículo científico. Esto también ayuda a que la información adquiera accesibilidad y permanencia en el largo plazo (a través de la revista científica y/o servicios de repositorios digitales). En el caso particular de este estudio, la caracterización de la amenaza permitió generar información útil para tomar medidas de manejo más efectivas (p.ej., se identificó los sitios y momentos en 
que se efectúa la pesca ilegal en el área marina protegida) (López-Garro et al., 2016).

Otro ejemplo de integración de esfuerzos en la Isla del Coco es el monitoreo de especies pelágicas a través de una de las compañías de buceo que visita frecuentemente el sitio (i.e., expediciones de buceo de $\sim 10$ días; $1-3$ veces al mes). Los datos se han tomado de forma continua desde el año 1991 y la compañía de turismo ha hecho disponibles los datos al sector académico. Con estos datos, se produjeron dos publicaciones científicas que demuestran un decline en las poblaciones de varias especies de tiburones y rayas (Sibaja-Cordero, 2008; White et al., 2015). En este caso, la coordinación ha sido principalmente entre la empresa turística y el sector académico, pero también con el sector de gestión del parque, el cual ha permitido mantener las estaciones de muestreo constantes, y ha logrado una regulación adecuada de la visitación turística. Dado que el objetivo principal de estas inmersiones no es el monitoreo, sino el turismo de recreación, es natural que existan debilidades metodológicas en estos muestreos (White et al., 2015). Por ejemplo, el conteo de individuos no se restringe a un campo visual de dimensiones constantes, hay riesgos de repetir individuos durante los conteos, y hay que hacer estimaciones visuales de números grandes de individuos (Roberto Cubero, Isaac Chinchilla, com. pers.). Sin embargo, el sector académico ha encontrado formas de compensar por estas debilidades y lograr extraer información relevante de este monitoreo (ver White et al., 2015; SibajaCordero, 2008). Los datos de este monitoreo señalan un decline de las poblaciones de varias especies de pelágicos en el área protegida (Sibaja-Cordero, 2008; White et al., 2015), lo cual es consistente con estudios paralelos en otros sitios del Pacífico Tropical Oriental (ver Hall \& Roman, 2013; Aires da Silva, LennertCody, Maunder, Momán-Verdesoto, \& Hinton, 2015; Jiménez, 2016). Aunque el sector académico podría generar muestreos enfocados a la toma más rigurosa de datos, lo cierto es que el costo de estos muestreos sería muy elevado (en comparación a los costos nulos actuales), y tampoco alcanzarían la misma resolución temporal de los monitoreos que se realizan hoy en día por el sector turismo.

Agendas particulares, institucionales, $y$ nacionales

Aun cuando se tienen objetivos comunes entre personas (p.ej., de conservación), existen agendas particulares o institucionales que dificultan trabajar de forma conjunta. Por ejemplo, en conservación y/o en la academia, se puede observar con cierta frecuencia una "territorialidad" sobre determinadas especies o sitios de estudio. Esta territorialidad puede entorpecer los esfuerzos de generar información y de lograr impacto en la conservación (Clark \& Brunner, 2002).

En parte, esta territorialidad se puede deber a que a menudo, las organizaciones e instituciones que trabajan en una región reciben financiamiento de la misma fuente. Sin embargo, los entes donantes están cada vez más interesados en la cooperación entre los receptores de financiamiento (Bode, Probert, Turner, Wilson, \& Venter, 2010). La idea detrás de esta nueva tendencia es que una meta de conservación (p.ej. recuperación de una especie en peligro de extinción) está fuera del alcance exclusivo de cualquier institución u organización, ya que ninguna tiene los recursos, la experiencia, los fondos y la autoridad suficiente para realizar dicha tarea por sí sola (Clark \& Brunner, 2002).

Es importante que las instituciones y organizaciones, consideren que a medida que fracasen distintas iniciativas de conservación, las especies focales se hacen más vulnerables a la extinción, y esto va en contra del fin último de la conservación (Clark \& Brunner, 2002). Al utilizar cooperativamente los recursos, las asociaciones pueden maximizar las posibilidades de recuperación de especies, y alcanzar objetivos de conservación en común (Clark \& Brunner, 2002). Existe evidencia que demuestra que la cooperación minimiza la ineficiencia logística, evita la redundancia de datos (Bode et al., 2010), y mejora la calidad de los programas de conservación (Clark \& Brunner, 2002). El 
personal a cargo de un área protegida puede promover que se dé esta cooperación entre los investigadores que la visitan, así como entre los distintos entes que financian la investigación en el sitio.

La Isla del Coco se caracteriza por contar con varias especies pelágicas residentes y migratorias (Sibaja-Cordero, 2008; Friedlander et al., 2012; Fourriére, Alvarado, Ayala-Bocos, \& Cortés, 2016), donde los tiburones son uno de los grupos más atractivos en términos de investigación científica (p.ej. Bessudo et al. 2011a; Bessudo et al., 2011b; Zanella, LópezGarro, Golfín-Duarte, \& Saenz, 2012; Arauz \& Hearn, 2014; White et al., 2015, Zanella, 2015; Zanella, López-Garro, Martínez, GolfínDuarte, \& Morales, 2015). Por facilidad logística, los estudios de tiburones en la región del Pacífico Tropical Oriental se han concentrado principalmente en los alrededores de las islas (Isla del Coco en Costa Rica, Archipiélago de Galápagos en Ecuador, e Isla Malpelo en Colombia). Estas áreas protegidas se podrían beneficiar si se lograra una cooperación más sólida entre los países y entre las organizaciones que generan datos en las diferentes islas de esta región. Lo anterior es indispensable, debido a que los objetos de conservación (i.e., especies pelágicas como los tiburones) no son particulares a uno de los sitios, sino que entran en zonas de pesca bajo la jurisprudencia de distintos países (ver Bessudo et al., 2011a; Bessudo et al., 2011b). Si se quiere caracterizar las poblaciones de estas especies, como el tiburón martillo (Sphyrna lewini), será necesario incrementar la escala espacial de los estudios, y esto solamente se podrá lograr a través de un esfuerzo internacional.

Una mayor cooperación podría incrementar la cantidad de especies que reciben atención científica. Por un lado, la cooperación permitiría incluir nuevos sitios de muestreo (p.ej., la Zona Económica Exclusiva de distintos países). Esto permitiría generar información sobre otras especies de tiburones (p.ej, sobre Carcharhinus falciformis), y no solamente sobre las especies que se agregan en las islas. También, al evitar una redundancia de esfuerzos, se podría incluir a otras especies además de tiburones, que también son amenazadas por la pesca (p.ej., Thunnus albacares y varias especies de picudos).

\section{Articulación entre el sector conservación y el sector de extracción.}

Una de las mayores amenazas para la biodiversidad de la Isla del Coco, y el Pacífico Tropical Oriental es la sobreexplotación pesquera (Sibaja-Cordero, 2008; Dapp et al., 2013; White et al., 2015; Arias \& Pressey, 2016; López-Garro et al., 2016). Sin embargo, esta es una amenaza para la cual se tiene poco conocimiento científico. En Costa Rica existen dos instituciones que tienen que ver con la generación de información, el uso, y la protección del recurso biológico marino. Una es el Sistema Nacional de Áreas de Conservación (SINAC) que gestiona la conservación y el manejo sostenible de la vida silvestre, y la otra es el Instituto Costarricense de Pesca y Acuicultura (INCOPESCA) que promueve el desarrollo pesquero bajo principios de sostenibilidad (INCOPESCA, 2018). Aunque tradicionalmente se maneja el concepto de que ambas instituciones (SINAC e INCOPESCA) tienen objetivos e intereses opuestos, lo cierto es que ambas comparten un interés en común (al menos en cuanto a los objetivos divulgados por la institución): garantizar la sostenibilidad del recurso pesquero a largo plazo.

La articulación de esfuerzos entre el SINAC y el INCOPESCA no es una tarea fácil; sin embargo, dicha articulación es imprescindible para generar estrategias de conservación del recurso pesquero y la biodiversidad marina a largo plazo. Ambas instituciones están interesadas en que el aprovechamiento del recurso pesquero sea sostenible a través del tiempo; por lo tanto, el aprovechamiento y la conservación del recurso están ligados y no se puede atender uno sin tomar en cuenta el otro. La discrepancia entre instituciones surge, aparentemente, cuando se favorece la explotación del recurso a corto plazo con consecuencias negativas en su conservación a largo plazo. Aunque normalmente esto se ve como un problema únicamente 
para el sector conservación, nótese que también es un problema que atenta contra la longevidad del sector pesquero a largo plazo; convirtiéndose así, en un problema socioeconómico de índole nacional. Para visualizar esta situación como un problema que atenta contra los objetivos de ambas instituciones, es prioridad que la información científica (que demuestra como el grado de explotación de un recurso va a definir el grado de aprovechamiento a largo plazo) sea el pilar de las decisiones que toma el sector extractivo. Esta es una tarea de divulgación científica que debería de ser prioridad para el sector académico y el sector conservación.

Bajo la idea de un objetivo común (conservación a largo plazo del recurso pesquero), es posible encontrar mecanismos para generar información en forma conjunta. Una posibilidad es que, a través de INCOPESCA, se logre generar información desde el punto de vista extractivo (p.ej, mediante observadores a bordo en la flota pesquera nacional e información de los sonares, de los dispositivos satelitales que utilizan las embarcaciones, y de los registros levantados por inspectores en puerto). El sector académico podría contribuir con ambas instituciones (SINAC e INCOPESCA) mediante el diseño de muestreos, análisis de estadísticas pesqueras, y recomendaciones técnicas que tomen en cuenta los intereses de ambas instituciones. Así, la información acerca de la extracción del recurso pesquero podría utilizarse con el propósito de que el recurso sea manejado bajo criterios de sostenibilidad a largo plazo (y no únicamente a corto plazo).

Esta articulación también podría facilitar el extraer información del sector pesquero internacional que opera en el Pacífico Tropical Oriental, y en la Zona Económica Exclusiva de Costa Rica (ver Arias \& Pressey, 2016). En el caso de Costa Rica, que posee una posición privilegiada en cuanto al recurso pesquero en el Pacífico Tropical Oriental (Jiménez, 2016), el lograr la articulación entre estas instituciones debería de ser un objetivo país.

Declaración de ética: los autores declaran que ambos están de acuerdo con esta publicación y que han hecho aportes que justifican su autoría; que no hay conflicto de interés de ningún tipo; y que han cumplido con todos los requisitos y procedimientos éticos y legales pertinentes. Todas las fuentes de financiamiento se detallan plena y claramente en la sección de agradecimientos. El respectivo documento legal firmado se encuentra en los archivos de la revista.

\section{AGRADECIMIENTOS}

Las ideas presentadas en este manuscrito son producto de un análisis posterior al proceso de elaboración de los Planes de Investigación del Área de Conservación Marina Cocos, proceso del cual participaron ambos autores y que fue, financiado por la Asociación Costa Rica por Siempre (ACRXS) y el Sistema Nacional de Áreas de Conservación (SINAC). Agradecemos las discusiones enriquecedoras que tuvimos durante dicho proceso con Mario Espinoza, Damián Martínez, Esteban Herrera, Oswaldo Rosero, Gustavo Induni, y Leonardo García. Además, agradecemos a las personas que de parte de las siguientes agrupaciones, ayudaron a comprender el contexto de conservación en la Isla del Coco: Área de Conservación Marina Cocos (ACMC) del SINAC, ACRXS, UnderSea Hunter, Misión Tiburón, Fins Attached, CREMA, FAICO, MarViva, Federación Costarricense de Pesca (FECOP), The Billfish Research Project, Centro de Investigación en Ciencias del Mar y Limnología (CIMAR), Instituto Costarricense de Pesca y Acuicultura (INCOPESCA), y la Universidad de Costa Rica. Finalmente, agradecemos a Jorge Cortés y a Beatriz Naranjo, por sugerir la escritura de este manuscrito para una edición especial de la Revista Biología Tropical. José Manuel Mora, Beatriz Naranjo, y un revisor anónimo hicieron observaciones que mejoraron la versión anterior del manuscrito.

\section{RESUMEN}

Introducción: En la práctica de la conservación biológica, a menudo se necesita de información científica 
para guiar las decisiones de manejo, pero esta información es especialmente escasa en áreas marinas protegidas. Las áreas marinas protegidas enfrentan el desafío de proteger especies pelágicas que se desplazan entre zonas protegidas de distintos países y zonas de pesca. En general, un obstáculo para generar la información que requieren las áreas protegidas es que comúnmente existe una disociación entre el sector académico y el sector de gestión, los cuales difieren en sus objetivos, forma de trabajo, y sistemas de recompensa. Esta situación se ve agravada en áreas marinas protegidas que se encuentran lejos de la costa, ya que los costos económicos y logísticos para realizar investigación son muy elevados. Objetivos: Utilizar el contexto del Parque Nacional Isla del Coco para ilustrar dificultades al momento de definir y recopilar la información necesaria para la gestión, y proponer posibles soluciones a este problema. Resultados: Para producir la información relevante, creemos que es necesario tomar en cuenta los siguientes aspectos: i) distinguir entre información biológica e información requerida para la gestión, ii) generar información sobre las amenazas a las especies, aún en ausencia de datos sobre las especies, iii) direccionar los planes de monitoreo, y iv) establecer una coordinación entre dos grupos de personas: las que frecuentan el área y las que están lejos del área en centros académicos. Además, debido a la naturaleza de la información que se requiere, y a la remotidad del sitio, se necesita de una articulación entre sectores (incluido el sector pesquero) para generar los datos. Conclusión: Parece indispensable lograr esta articulación entre sectores, lo cual conlleva grandes retos. Aún así, esta opción parece ser más viable desde el punto de vista logístico y económico que el intentar generar esta información desde una plataforma exclusivamente académica. Además, dicha articulación parece ser la única forma de generar información que es requerida para regular el manejo pesquero; por ejemplo la caracterización adecuada del esfuerzo de captura y de la biomasa extraída.

Palabras clave: investigación para la conservación, amenazas a la biodiversidad, monitoreo biológico, políticas de conservación, sobrepesca, especies pelágicas.

\section{REFERENCIAS}

Aires da Silva, A., Lennert-Cody, C., Maunder, M. N., Román-Verdesoto, M., \& Hinton, M. G. (2015). Stock status indicators for silky sharks in the Eastern Pacific Ocean 6th Meeting the IATTC Scientific Advisor Meeting (Document SAC 06 08b). California: InterAmerican Tropical Tuna Commission.

Anderson, C. N. K., Hsieh, C., Sandin, S. A., Hewitt, R., Hollowed, A., Beddington, J., May, R. M., \& Sugihara, G. (2008). Why fishing magnifies fluctuations in fish abundance. Nature, 452, 835-839.

Arauz, H., \& Hearn, A. (2014). Movimientos migratorios de tiburones marcados en la Isla del Coco.
Memoria: Taller Interinstitucional de Implementación de Medidas CITES para el Tiburón Martillo, Tiburón Punta Blanca Oceánico y la Manta Raya. Heredia, Costa Rica.

Arias, A., \& Pressey, R. L. (2016). Combating Illegal, Unreported, and Unregulated Fishing with Information: A Case of Probable Illegal Fishing in the Tropical Eastern Pacific. Frontiers in Marine Science, 3, 1-4.

Arias, A., Pressey, R. L., Jones, R. E., Álvarez-Romero, J. G., \& Cinner J. E. (2014). Optimizing enforcement and compliance in offshore marine protected areas: a case study from Cocos Island, Costa Rica. Oryx, 50, 18-26. DOI:http://dx.doi.org/10.1017/ S0030605314000337.

Bakker, V. J., \& Doak, D. F. (2009). Population viability management: ecological standards to guide adaptive management for rare species. Frontiers in Ecology and the Environment, 7, 158-165.

Balmford, A., \& Cowling, R. M. (2006). Fusion or Failure? The Future of Conservation Biology. Conservation Biology, 20, 692-695.

Bessudo, S., Soler, G., Klimley, A. P., Ketchum, J. T., Arauz, R., \& Hearn, A. (2011a.) Residency of the scalloped hammerhead shark (Sphyrna lewini) at Malpelo Island and evidence of migration to other islands in the Eastern Tropical Pacific. Environmental Biology Fishes, 91, 165-176.

Bessudo, S., Soler, G., Klimley, A. P., Ketchum, J. T., Arauz, R., Hearn, A., Guzmán, A., \& Calmenettes, B. (2011b). Vertical and horizontal movements of the scalloped hammerhead shark (Sphyrna lewini) around Malpelo and Cocos islands (Tropical Eastern Pacific) using satellite telemetry. Boletín de investigaciones marina y costeras, 40, 91-106.

Bode, M., Probert, W., Turner, W. R., Wilson, K. A., \& Venter, O. (2010). Conservation Planning with multiple organizations and objectives. Conservation Biology, 25, 295-304.

Bonney, R., Shirk, J. L., Phillips, T. B., Wiggins, A., Ballard, H. L., Miller-Rushing, A. J., \& Parrish, J. K. (2014). Next step for citizen science. Science, 343, 1436-1437.

Bottrill, M. C., Joseph, L. N., Carwardine, J., Bode, M., Cook, C., Game, E. T., Grantham, H., Kark, S., Linke, S., McDonald-Madden, E., \& Pressey, R. L. (2008). Is conservation triage just smart decision making? Trends in Ecology \& Evolution, 23, 649-654.

Camhi, M. D., Pikitch, E. K., \& Babcock, E. A. (Ed.) (2018). Sharks of the open ocean: biology, fisheries and conservation. Oxford: Blackwell Publishing Ltd.

Clark, T. W., \& Brunner, R. D. (2002). Making partnerships work in endangered species conservation: an 
introduction to the decision process. Endangered Species UPDATE, 13, 1-5.

Dapp, D., Arauz, R., Spotila, J. R., \& O’Connor, M. P. (2013). Impact of Costa Rican longline fishery on its bycatch of sharks, stingrays, bony fish and olive ridley turtles (Lepidochelys olivacea). Journal of Experimental Marine Biology and Ecology, 448, 228-239.

Ellstrand, N. C, Biggs, D., Kaus, A., Lubinsky, P., McDade, L. A., Preston, K., Prince, L. M., Regan, H. M., Rorive, V., Ryder, O. A., \& Schierenbeck, K. A. (2010). Got hybridization? A multidisciplinary approach for informing science policy. BioScience, 60, 384-388.

Fourriére, M., Alvarado, J. J., Ayala-Bocos, A., \& Cortés, J. (2016). Updated checklist and analysis of completeness of the marine fish fauna of Isla del Coco, Pacific of Costa Rica. Marine Biodiversity, 47, 813-821.

Fox, H. E., Mascia, M. B., Basurto, X., Costa, A., Glew, L., Heinemann, D., Karrer, L. B., Lester, S. E., Lombana, A. V., Pomeroy, R. S., Recchia, C. A., Roberts, C. M., Sanchirico, J. N., Pet-Soede, L., \& White, A.T. (2012). Reexamining the science of marine protected areas: linking knowledge to action. Conservation Letters, 5, 1-10.

Friedlander, A. M., Zgliczynski, B. J., Ballesteros, E., Aburto-Oropeza, O., Bolaños, A., \& Sala, E. (2012). The shallow-water fish assemblage of Isla del Coco National Park, Costa Rica: Structure and patterns in an isolated, predator-dominated ecosystem. Revista Biología Tropical, 60, 321-338.

Gerber, L. R., \& Schultz, C. B. (2001). Authorship and the Use of Biological Information in Endangered Species Recovery Plans. Conservation Biology, 15, $1308-1314$

Gonzáles-Andrés, C., Lopes, P. F. M, Cortés, J., SánchezLizano, J. L., \& Pennino, M. G. (2016). Abundance and Distribution Patterns of Thunnus albacares in Isla del Coco National Park through Predictive Habitat Suitability Models. PLoS ONE, 11, e 0168212.

Hall, M., \& Roman, M. (2013). Bycatch and non-tuna in the tropical tuna purse-seine fisheries of the world (Techinical paper \# 568). Roma: FAO-Fisheries and Aquaculture.

Hector, A., \& Bagchi, R. (2007). Biodiversity and ecosystem multi-functionality. Nature, 448, 188-90.

Hinton, M., Maunder, M., Vogel, N., Olson, R., Lennert, C., Aires-da-Silva, A., \& Hall, M. (2014). Stock status indicators for fisheries of the Eastern Pacific Ocean (Stock Assessment Report). California: InterAmerican Tropical Tuna Commission.

Huenneke, L. F. (1995). Involving academic scientists in conservation research: perspectives of a plant ecologist. Ecological Applications, 5, 209-214.
Hyder, K., Townhill, B., Anderson, L. G., Delany, J., \& Pinnegar, J. K. (2015). Can citizen science contribute to the evidence-based that underpins marine policy? Marine Policy, 59, 112-120.

Instituto Costarricense de Pesca y Acuicultura (INCOPESCA). (2018). ¿Quiénes somos?. Puntarenas, Costa Rica. INCOPESCA (Descargado: dic 6, 2018, https:// www.incopesca.go.cr/acerca_incopesca/)

Jiménez, J. A. (2016). El Domo Térmico de Costa Rica: Un oasis de productividad frente a las costas del Pacifico Centroamericano. San José: Fundación MarViva.

Karam-Gemael, M., Loyala, R., Penha, J., \& Izzo, T. (2018). Poor alignment of priorities between scientists and policymakers highlights the need for evidence-informed conservation in Brazil. Perspective in Ecology and Evolution, 16, 125-132.

Lande, R., Engen, S., \& Sæther, B. E. (2003). Stochastic Population Dynamics in Ecology and Conservation. New York: Oxford University Press.

Laurance, W. F., Koster, H., Grooten, M., Anderson, A. B. Zuidema, P. A. Zwick, S., Zagt, R. J. Lynam, A. J., Linkie, M., \& Anten, N. P. R. (2012). Making conservation research more relevant for conservation practitioners. Biological Conservation, 153, 164-168.

Law, R. (2000). Fishing, selection, and phenotypic evolution. ICES Journal of Marine Science, 57, 659-668.

Lawton, J. H. (1999). Are there general laws in ecology? Oikos, 84, 177-192.

Legg, C. J., \& Nagy, L. (2006). Why most conservation monitoring is, but need not be, a waste of time. Journal of Environmental Management, 78, 194-199.

López-Garro, A., Zanella, I., Martínez, F., Golfín-Duarte, G., \& Pérez-Montero, M. (2016). La pesca ilegal en el Parque Nacional Isla del Coco, Costa Rica. Revista Biología Tropical, 64, (Supl. 1), 249-262.

Lundquist, C. J., \& Granek, E. F. (2005). Strategies for successful marine conservation: integrating socioeconomic, political, and scientific factors. Conservation Biology, 19, 1771-1778.

Magurran, A. E., Baille, S. R., Buckland, S. T., Dick, J. M., Elston, D. A., Scott, E. M, Smith, R. I, Somerfield, P. J., \& Watt, A. D. (2010). Long-term datasets in biodiversity research and monitoring: assessing change in ecological communities through time. Trends in Ecology and Evolution, 25, 574-582.

Maunder, M. N., Sibert, J. R., Fonteneau, A., Hampton, J., Kleiber, P., \& Harley, S. J. (2006). Interpreting catch per unit effort data to assess the status of individual stocks and communities. ICES Journal of Marine Science, 63, 1373-1385. 
Newing, H. (2011). Conducting Research in Conservation: Social science methods and practice. Oxford, UK: Routledge.

Nichols, J. D., \& Williams, B. K. (2006). Monitoring for conservation. Trends in Ecology and Evolution, 21, 668-673.

Parrish, J. D, Barun, D. P., \& Unnasch, R. S. (2003). Are We Conserving What We Say We Are? Measuring Ecological Integrity within Protected Areas. BioScience, 53, 851-860.

Polasky, S., Carpenter, S. R., Folke, C., \& Keeler, B. (2011). Decision making under great uncertainty: environmental management in an era of global change. Trends in Ecology and Evolution, 26, 398-404.

Possingham, H. P., Andelman, S. J., Noon, B. R., Trombulak, S., \& Pulliam, H. R. (2000). Making smart conservation decisions. Conservation Biology: Research Priorities for the Next Decade, 23, 225-244.

Primack, R. B. (2006). Essentials for conservation biology. Massachusetts: Sinauer Associates.

Pullin, A. S., Knight, T. M., Stone, D. A., \& Charman, K. (2004). Do conservation managers use scientific evidence to support their decision-making? Biological Conservation, 119, 245-252.

Ricklefs, R. E. (2008). Disintegration of the Ecological Community. American Naturalist, 172, 741-750.

Robertson, D. P., \& Hull, B. (2001). Beyond biology: toward a more public ecology for conservation. Conservation Biology, 15, 970-979.

Robertson, D. R., \& Cramer, K. L. (2009). Shore fishes and biogeographic subdivisions of the Tropical Eastern Pacific. Marine Ecology Progress Series, 380, 1-17.

Rojas, J. R., Campos, J., Segura, A., Moisés, M., Campos, R., \& Rodríguez, O. (2000). Shark fisheries in Central America: a review and update. UNICIENCIA, $17,49-56$.

Salafsky, N., Margoluis, R., \& Redford, K. H. (2002). Improving the practice of conservation: a conceptual framework and research agenda for conservation science. Conservation Biology, 16, 1469-1479.

Salafsky, N., Salzer, D., Ervin, J., Boucher, T., \& Ostile, W. (2003). Conventions for defining, naming, measuring, combining, and mapping threats in conservation: an initial proposal for a standard system. Conservation Measures Partnership. Washington, USA. Recuperado de http://citeseerx.ist.psu.edu/viewdoc/download doi $=10.1 \cdot 1 \cdot 525.8866 \&$ rep $=$ rep $1 \&$ type $=$ pdf

Sibaja-Cordero, J. A. (2008). Tendencias espacio-temporales de los avistamientos de fauna marina en los buceos turísticos (Isla del Coco, Costa Rica). Revista Biología Tropical, 56, 113-132.
Sibert, J., Hampton, J., Kleiber, P., \& Maunder, M. (2006). Biomass, size, and trophic status of top predators in the Pacific Ocean. Science, 314, 1773-1776.

Soulé, M. E. (1985) What is Conservation Biology?: A new synthetic discipline addresses the dynamics and problems of perturbed species, communities, and ecosystems. BioScience, 35, 727-734.

Sutherland, W. J., Fleishman. E., Mascia, M. B., Pretty, J., \& Rudd, M. A. (2011). Methods for collaboratively identifying research priorities and emerging issues in science and policy. Methods in Ecology and Evolution, 2, 238-247.

Trippel, E. A. (1995). Age at maturity as a stress indicator in fisheries. Bioscience, 45, 759-771.

Verner, J. (1992). Data Needs for Avian Conservation Biology: Have We Avoided Critical Research? The Condor, 94, 301-303.

Vos, P., Meelis, E., \& Keurs, W. J. T. (2000). A framework for the design of ecological monitoring programs as a tool for environmental and nature management. Environmental Monitoring and Assessment, 61, 317-344.

Ward-Paige, C. A. (2017). A global overview of sheer sanctuary regulations and their impact on shark fisheries. Marine Policy, 82, 87-97.

Watson, J. T., Essington, T. E., Lennert-Cody, C. E., \& Hall, M. A. (2009). Trade-offs in the design of fishery closures: management of silky shark bycatch in the Eastern Pacific Ocean tuna fishery. Conservation Biology, 23, 626-635.

White, E. R., Myers, M. C., Flemming, J. M., \& Baum, J. K. (2015). Shifting elasmobranch community assemblage at Cocos Island: an isolated marine protected area. Conservation Biology, 29, 1186-1197.

Whoriskey, S., Arauz, R., \& Baum, J. K. (2011). Potential impacts of emerging mahi-mahi fisheries on sea turtle and elasmobranch bycatch species. Biological Conservation, 144, 1841-1849.

Zanella, I. (2015). Conservation of scalloped hammerhead shark (Sphyrna lewini) and its critical habitat in Golfo Dulce, Costa Rica. 19th Annual Scientific Conference of the European Elasmobranch Association. Peniche, Portugal.

Zanella, I., López-Garro, A., Golfín-Duarte, G., \& Saenz, J. (2012). Abundancia, tamaño y estructura poblacional del tiburón punta blanca de arrecife, Triaenodon obesus (Carcharhiniformes: Carcharhinidae), en Bahía Chatham, Parque Nacional Isla del Coco, Costa Rica. Revista Biología Tropical, 60, 339-346.

Zanella, I., López-Garro, A., Martínez, F., Golfín-Duarte, G., \& Morales, K. (2015). Poblaciones de tiburones en las bahías Chatham y Wafer del Parque Nacional Isla del Coco, Costa Rica. Revista Biología Tropical, 64, S177-S186. 